\title{
EQUIVALENCE OF BANACH SPACE-VALUED ORNSTEIN-UHLENBECK PROCESSES
}

\author{
ZDZISLAW BRZEŹNIAK ${ }^{1}$ AND JAN VAN NEERVEN ${ }^{2}$
}

\begin{abstract}
Let $\mu_{A}$ be the law of the Ornstein-Uhlenbeck process that solves the equation

$$
d \xi_{t}=A \xi_{t} d t+d W(t), \quad \xi_{0}=0 \text { almost surely, }
$$

where $A$ is a generator of a $C_{0}$-semigroup on a Banach space $B$ and $W(t), t \geqslant 0$, is a cylindrical Wiener process on a Hilbert subspace $H$ of $B$. For two 'drift' operators $A_{0}$ and $A_{1}$ we study sufficient and necessary conditions for the measures $\mu_{A_{0}}$ and $\mu_{A_{1}}$ to be equivalent.
\end{abstract}

\section{INTRODUCTION}

Suppose $\mathscr{S}_{0}=\left\{S_{0}(t)\right\}_{t \geqslant 0}$ and $\mathscr{S}_{1}=\left\{S_{1}(t)\right\}_{t \geqslant 0}$ are two $C_{0}$-semigroups of bounded linear operators on a separable real Hilbert space $H$, which is continuously embedded into some real Banach space $B$. Let $\xi^{0}=\left\{\xi_{t}^{0}\right\}_{t \geqslant 0}$ and $\xi^{1}=\left\{\xi_{t}^{1}\right\}_{t \geqslant 0}$ be $B$-valued Ornstein-Uhlenbeck processes with drifts $\mathscr{S}_{0}$ and $\mathscr{S}_{1}$, respectively; see Section 4 for the definition. One could think of a $B$-valued Ornstein-Uhlenbeck process with drift $\mathscr{S}$ as a $B$-valued solution of the stochastic differential equation (SDE)

$$
d \xi_{t}=A \xi_{t} d t+d W_{t}, \quad \xi_{0}=0 \text { almost surely }
$$

where $A$ is the generator of $\mathscr{S}$ and $d W$ is $H$-valued white noise.

In this paper we study the problem under which conditions the processes $\xi^{0}$ and $\xi^{1}$ are 'equivalent'. In order to give the notion of 'equivalence' a precise mathematical meaning, we use the concept of an Ornstein-Uhlenbeck measure. A probability measure $\nu$ on the product space $B^{[0, T]}$ will be called a Ornstein-Uhlenbeck measure with drift $\mathscr{S}$ if the canonical process on $\left(B^{[0, T]}, \nu\right)$ defined by

$$
\xi_{t}(\omega)=\omega(t) \quad\left(t \in[0, T], \omega \in B^{[0, T]}\right),
$$

is a $B$-valued Ornstein-Uhlenbeck process with drift $\mathscr{S}$. To every $B$-valued OrnsteinUhlenbeck process with drift $\mathscr{S}$ there corresponds a unique canonical Ornstein-Uhlenbeck process with drift $\mathscr{S}$, and thus a unique Ornstein-Uhlenbeck measure $\nu$ on $B^{[0, T]}$. We now define two Ornstein-Uhlenbeck processes $\xi^{0}$ and $\xi^{1}$ to be equivalent if the corresponding Ornstein-Uhlenbeck measures $\nu_{0}$ and $\nu_{1}$ are equivalent, i.e. if $\nu_{0}$ and $\nu_{1}$ are absolutely continuous with respect to each other.

Denoting by $D(A)$ the domain of an operator $A$, and by $\|\cdot\|_{\mathscr{L}_{2}(H)}$ the Hilbert-Schmidt norm of an operator on $H$, our main results can be formulated as follows:

1991 Mathematics Subject Classification. 60G15, 60H15, 60B05, 47D03.

Key words and phrases. Ornstein-Uhlenbeck process, (equivalence of) Gaussian measures on a Banach space, Feldman-Hájek Theorem, $C_{0}$-semigroups on Banach spaces.

${ }^{1}$ Part of this work was supported by the Deutsche Forschungs Gemeinschaft (DFG).

2 This research has been made possible by a fellowship of the Royal Netherlands Academy of Arts and Sciences and by an EPSRC Visiting Fellowship GR/L 60876 at the University of Hull . 
Theorem 1.1. Let $\xi^{0}$ and $\xi^{1}$ be two B-valued Ornstein-Uhlenbeck processes, with drifts $\mathscr{S}_{0}$ and $\mathscr{S}_{1}$ respectively, and let $\nu_{0}$ and $\nu_{1}$ be the associated Ornstein-Uhlenbeck measures on $B^{[0, T]}$. Consider the following three conditions:

(i) $D\left(A_{0}\right)=D\left(A_{1}\right)$, for each $t \in(0, T]$ the linear operator $h \mapsto\left(A_{0}-A_{1}\right) S_{0}(t) h$, defined on the common domain of $A_{0}$ and $A_{1}$, extends to a bounded operator on $H$, and

$$
\int_{0}^{T}\left\|\left(A_{0}-A_{1}\right) S_{0}(s)\right\|_{\mathscr{L}_{2}(H)}^{2} d s<\infty
$$

(ii) $D\left(A_{0}\right)=D\left(A_{1}\right)$, for each $t \in(0, T]$ the linear operator $h \mapsto\left(A_{1}-A_{0}\right) S_{1}(t) h$, defined on the common domain of $A_{0}$ and $A_{1}$, extends to a bounded operator on $H$, and

$$
\int_{0}^{T}\left\|\left(A_{1}-A_{0}\right) S_{1}(s)\right\|_{\mathscr{L}_{2}(H)}^{2} d s<\infty
$$

(iii) The measures $\nu_{0}$ and $\nu_{1}$ are equivalent.

Then (i) and (ii) are equivalent and imply (iii).

In the converse direction, with the same notation as in Theorem 1.1 we have:

Theorem 1.2. If $\nu_{0}$ and $\nu_{1}$ are equivalent, then $D\left(A_{0}\right)=D\left(A_{1}\right)$. If furthermore for each $t \in(0, T]$ the operator $\left(A_{0}-A_{1}\right) S_{0}(t)$, defined on the common domain of $A_{0}$ and $A_{1}$, has a bounded extension to $H$, such that

$$
\int_{0}^{T}\left\|\left(A_{0}-A_{1}\right) S_{0}(t)\right\| d t<\infty
$$

then for each $t \in(0, T]$ the operators $\left(A_{0}-A_{1}\right) S_{0}(t)$ and $\left(A_{1}-A_{0}\right) S_{1}(t)$ extend to HilbertSchmidt operators on $H$ and

$$
\int_{0}^{T}\left\|\left(A_{0}-A_{1}\right) S_{0}(s)\right\|_{\mathscr{L}_{2}(H)}^{2} d s<\infty \quad \text { and } \quad \int_{0}^{T}\left\|\left(A_{1}-A_{0}\right) S_{1}(s)\right\|_{\mathscr{L}_{2}(H)}^{2} d s<\infty .
$$

For certain selfadjoint elliptic operators, equivalence of Hilbert space-valued OrnsteinUhlenbeck semigroups was first studied by Kozlov [5]. His work was extended by Peszat [9], who considered the case where $B=H$, assuming that

$$
\int_{0}^{T}\left\|S_{j}(t)\right\|_{\mathscr{L}_{2}(H)}^{2} d t<\infty \quad(j=0,1)
$$

along with somewhat stronger integrability conditions on the semigroups $\mathscr{S}_{0}$ and $\mathscr{S}_{1}$; see also [3, Chapter 10]. Equivalence of $B$-valued Ornstein-Uhlenbeck processes corresponding to generators of analytic semigroups has been considered by Röckle [11].

Theorems 1.1 and 1.2 are proved in Section 4 and depend on some perturbation results for $C_{0}$-semigroups and equivalence results for Gaussian measures, which are presented in Sections 2 and 3 respectively.

\section{A perturbation Result}

In this preliminary section we will prepare two perturbation results for $C_{0}$-semigoups that will be needed later on. The first of these, which was also used in [9], may be found in [4, Theorem 3.4.1, p. 399; Corollary 1, p. 400, Theorem 13.5.2, p. 409]. We assume the reader to be familiar with the elementary concepts of semigroup theory as can be found in e.g. [8], [4].

Proposition 2.1. Suppose that $A_{0}$ and $A_{1}$ are the generators of $C_{0}-$ semigroups $\mathscr{S}_{0}$ and $\mathscr{S}_{1}$ on a Banach space $X$ such that the following three conditions hold: 
(i) $D\left(A_{0}\right)=D\left(A_{1}\right)$;

(ii) For each $t \in(0, T]$ the linear operator $\left(A_{0}-A_{1}\right) S_{0}(t)$ has a bounded extension to $X$;

(iii) $\int_{0}^{T}\left\|\left(A_{0}-A_{1}\right) S_{0}(t)\right\| d t<\infty$,

Then, symmetrically, we have

(ii)' For each $t \in(0, T]$ the linear operator $\left(A_{1}-A_{0}\right) S_{1}(t)$ has a bounded extension to $X$; (iii) $\int_{0}^{T}\left\|\left(A_{1}-A_{0}\right) S_{1}(t)\right\| d t<\infty$.

Moreover, we have the following 'variation of constants' formula:

$$
S_{1}(t) x=S_{0}(t) x+\int_{0}^{t} S_{0}(t-s)\left(A_{1}-A_{0}\right) S_{1}(s) x d s, \quad x \in X .
$$

Let $X$ be a Banach space and let $\mathscr{I} \subset \Lambda(X)$ be an operator ideal, i.e. $\mathscr{I}$ is a linear subspace of $\Lambda(X)$ which is a Banach space with respect to a norm $\|\cdot\|_{\mathscr{I}}$ which is stronger than the operator norm on $\Lambda(X)$, such that

$$
\left\|S_{0} T S_{1}\right\|_{\mathscr{I}} \leqslant\left\|S_{0}\right\|\|T\|_{\mathscr{I}}\left\|S_{1}\right\|, \quad S_{0}, S_{1} \in \Lambda(X), T \in \mathscr{I} .
$$

Proposition 2.2. Suppose $A_{0}$ and $A_{1}$ are generators of $C_{0}-$ semigroups $\mathscr{S}_{0}$ and $\mathscr{S}_{1}$ on a Banach space $X$ such that $D\left(A_{0}\right)=D\left(A_{1}\right)$. Let $\mathscr{I}$ be an operator ideal in $\Lambda(X)$. Then the following assertions are equivalent:

(i) For each $t \in(0, T]$ the operator $\left(A_{0}-A_{1}\right) S_{0}(t)$ extends to an element of $\mathscr{I}$ and we have

$$
\int_{0}^{T}\left\|\left(A_{0}-A_{1}\right) S_{0}(t)\right\|_{\mathscr{I}}^{2} d t<\infty
$$

(ii) For each $t \in(0, T]$ the operator $\left(A_{0}-A_{1}\right) S_{1}(t)$ extends to an element of $\mathscr{I}$ and we have

$$
\int_{0}^{T}\left\|\left(A_{0}-A_{1}\right) S_{1}(t)\right\|_{\mathscr{I}}^{2} d t<\infty .
$$

Proof. We prove that (i) implies (ii); the converse implication follows by symmetry. By (i), the fact that $\|\cdot\|_{\mathscr{I}}$ is stronger than the operator norm and by the Cauchy-Schwartz inequality,

Hence by Proposition 2.1,

$$
\int_{0}^{T}\left\|\left(A_{0}-A_{1}\right) S_{0}(s)\right\| d s<\infty .
$$

$$
\int_{0}^{T}\left\|\left(A_{0}-A_{1}\right) S_{1}(s)\right\| d s<\infty .
$$

Therefore, by [4, Lemma 13.3.5, p. 395] for all $t \in[0, T]$ and $x \in X$ we have

$$
\left(A_{0}-A_{1}\right) \int_{0}^{t} S_{0}(t-s)\left(A_{1}-A_{0}\right) S_{1}(s) x d s=\int_{0}^{t}\left(A_{0}-A_{1}\right) S_{0}(t-s)\left(A_{1}-A_{0}\right) S_{1}(s) x d s .
$$

By this identity, (2.1), and the Young inequality,

$$
\begin{aligned}
\int_{0}^{T} & \left\|\left(A_{1}-A_{0}\right)\left(S_{1}(t)-S_{0}(t)\right)\right\|_{\mathscr{I}}^{2} d t \\
& \leqslant \int_{0}^{T}\left(\int_{0}^{t}\left\|\left(A_{1}-A_{0}\right) S_{0}(t-s)\right\|_{\mathscr{I}}\left\|\left(A_{1}-A_{0}\right) S_{1}(s)\right\| d s\right)^{2} d t \\
& \leqslant \int_{0}^{T}\left\|\left(A_{1}-A_{0}\right) S_{0}(s)\right\|_{\mathscr{I}}^{2} d s\left(\int_{0}^{T}\left\|\left(A_{1}-A_{0}\right) S_{1}(s)\right\| d s\right)^{2}<\infty .
\end{aligned}
$$


By combining this estimate with (i), condition (ii) immediately follows.

\section{Equivalence of Gaussian measures}

In this section we briefly recall some well-known facts concerning (cylindrical) Gaussian measures. For more details we refer to [14], [15], [12], [13], [6].

Let $E$ be a real locally convex topological vector space, with topological dual $E^{\prime}$. A subset $C$ of $E$ is said to be a cylindrical set if it is contained in the algebra $\mathscr{C}(E)$ of subsets of $E$ generated by all sets of the form $\left\{x \in E:\left(\left\langle x, x_{1}^{\prime}\right\rangle, \ldots,\left\langle x, x_{n}^{\prime}\right\rangle\right) \in B\right\}$ for some $n \geqslant 1, x_{1}^{\prime}, \ldots, x_{n}^{\prime} \in E^{\prime}$, and Borel set $B \subset \mathbb{R}^{n}$. A centered cylindrical Gaussian measure on $E$ is a finitely additive set function $\mu$ on $\mathscr{C}(E)$, whose images under the maps $x \mapsto\left(\left\langle x, x_{1}^{\prime}\right\rangle, \ldots,\left\langle x, x_{n}^{\prime}\right\rangle\right)$ are $\sigma$-additive Gaussian measures on $\mathbb{R}^{n}$.

If $F$ is another real locally convex topological vector space, and if $T: E \rightarrow F$ is a continuous linear transformation, then the image $T(\mu):=\mu \circ T^{-1}$ of a centered cylindrical Gaussian measure on $E$ is a centered cylindrical Gaussian measure on $F$.

Let $H$ be a real Hilbert space. By $\gamma_{H}$ we denote the standard centered cylindrical Gaussian measure on $H$, i.e. the centered cylindrical Gaussian measure on $H$ whose image under any of the maps $g \mapsto\left(\left[g, h_{1}\right]_{H}, \ldots,\left[g, h_{n}\right]_{H}\right)$ is the standard Gaussian measure on $\mathbb{R}^{n}$.

A continuous linear operator $Q \in \Lambda\left(E^{\prime}, E\right)$ is called positive if $\left\langle Q x^{\prime}, x^{\prime}\right\rangle \geqslant 0$ for all $x^{\prime} \in E^{\prime}$, and symmetric if $\left\langle Q x^{\prime}, y^{\prime}\right\rangle=\left\langle Q y^{\prime}, x^{\prime}\right\rangle$ for all $x^{\prime} \in E^{\prime}$ and $y^{\prime} \in E^{\prime}$. To every positive symmetric operator $Q \in \Lambda\left(E^{\prime}, E\right)$ one can associate a real Hilbert space $H_{Q}$ in the following way. On the range of $Q$ one has a well-defined inner product $[\cdot, \cdot]_{H}$ given by

$$
\left[Q x^{\prime}, Q y^{\prime}\right]:=\left\langle Q x^{\prime}, y^{\prime}\right\rangle \quad\left(x^{\prime}, y^{\prime} \in E^{\prime}\right) .
$$

Denote by $H_{Q}$ the Hilbert space completion of range $Q$ with respect to this inner product; this Hilbert space is called the reproducing kernel Hilbert space (RKHS) associated with $Q$. If $E$ is qusi-complete, then the inclusion mapping from range $Q$ into $E$ has a continuous extension to an injective linear map $i: H_{Q} \rightarrow E$. In this way, the pair $\left(i, H_{Q}\right)$ becomes a Hilbert subspace of $E$. Moreover, upon identifying $H_{Q}$ with its dual in the natural way, we then have the operator identity $Q=i \circ i^{\prime}$.

Conversely, if $(i, H)$ is a real Hilbert subspace of $E$ (i.e. $i$ is a continuous injective linear map from some real Hilbert space $H$ into $E)$, then $Q:=i \circ i^{\prime} \in \mathscr{L}\left(E^{\prime}, E\right)$ is positive and symmetric, and its RKHS equals $H$.

The relationship between centered cylindrical Gaussian measures and positive symmetric operators in described in the following well-known result.

Proposition 3.1. Let $E$ be a real locally convex topological vector space.

(i) Let $H$ be a real Hilbert space and let $T \in \Lambda(H, E)$. The image cylindical measure $\mu:=T\left(\gamma_{H}\right)$ is a centered cylindrical Gaussian measure on E, whose Fourier transform is given by

$$
\int_{E} \exp \left(i\left\langle x, x^{\prime}\right\rangle\right) d \mu(x)=\exp \left(-\frac{1}{2}\left\langle T \circ T^{\prime} x^{\prime}, x^{\prime}\right\rangle\right) \quad\left(x^{\prime} \in E^{\prime}\right) .
$$

Moreover, the RKHS $H_{Q}$ associated with the positive symmetric operator $Q=T \circ T^{\prime} \in$ $\Lambda\left(E^{\prime}, E\right)$ is the orthogonal complement in $H$ of the kernel of $T$. As such, $H_{Q}$ can be identified with range $T$, the inner product being given by

$$
[\mathrm{Tg}, \mathrm{Th}]_{H_{Q}}=[\mathrm{Pg}, \mathrm{Ph}]_{H},
$$

where $P$ is the orthogonal projection in $H$ onto $(k e r T)^{\perp}$. 
(ii) If $E$ is quasi-complete and $Q \in \Lambda\left(E^{\prime}, E\right)$ is positive and symmetric, and if $\mu$ is a centered cylindrical Gaussian measure on E with Fourier transform

$$
\int_{E} \exp \left(i\left\langle x, x^{\prime}\right\rangle\right) d \mu(x)=\exp \left(-\frac{1}{2}\left\langle Q x^{\prime}, x^{\prime}\right\rangle\right) \quad\left(x^{\prime} \in E^{\prime}\right),
$$

then $\mu=i\left(\gamma_{H}\right)$, where $H$ is the RKHS of $Q$ and $i: H \hookrightarrow E$ is the natural embedding.

Let $E$ be a real locally convex topological vector space. A measure $\mu$ on the $\sigma$-algebra $\sigma(\mathscr{C}(E))$ generated by the algebra $\mathscr{C}(E)$ is called a (centered) Gaussian measure on $E$ if for all $x^{\prime} \in E^{\prime}$ the image measure $\left\langle\mu, x^{\prime}\right\rangle:=\mu \circ\left(x^{\prime}\right)^{-1}$ is a (centered) Gaussian Borel measure on $\mathbb{R}$. A continuous linear operator $T \in \Lambda(H, E)$ is said to be $\gamma_{H}$-radonifying (or, if no confusion is possible, radonifying) if the image cylindrical measure $T\left(\gamma_{H}\right)$ has a (necessarily unique) countably additive extension to a Gaussian measure on $E$.

If $E$ is separable, then $\sigma(\mathscr{C}(E))$ is just the Borel $\sigma$-algebra of $E$, but in general $\sigma(\mathscr{C}(E))$ is much smaller.

The following three examples of radonifying operators will be of importance:

- If $\mu$ is a centered Gaussian measure on $E$ with RKHS $H$, then the inclusion map $i: H \rightarrow E$ is radonifying, and we have $i\left(\gamma_{H}\right)=\mu$.

- If $H$ and $E$ are Hilbert spaces, then $T \in \Lambda(\mathscr{H}, E)$ is radonifying if and only if $T$ is a Hilbert-Schmidt operator.

- If $G$ and $H$ are Hilbert spaces and $S \in \Lambda(G, H)$ and $T \in \Lambda(H, E)$ are continuous linear operators, then $T \circ S$ is radonifying whenever $T$ is radonifying [1], [10].

Two measures $\mu_{0}$ and $\mu_{1}$ defined on the same $\sigma$-algebra are said to be equivalent, notation $\mu_{0} \sim \mu_{1}$, if they are absolutely continuous with respect to each other, i.e. if $\mu_{0} \ll \mu_{1}$ and $\mu_{1} \ll \mu_{0}$. They are said to be singular, notation $\mu_{0} \perp \mu_{1}$, if there exist disjoint measurable sets $B_{0}$ and $B_{1}$ such that $\mu_{0}\left(B_{0}\right)=\mu_{1}\left(B_{1}\right)=1$. Two Gaussian measures on a real locally convex topological vector space $E$ are either equivalent or singular. This result is due Vakhania and Tarieladze [14], who also give necessary and sufficent conditions for equivalence. In case both $\mu_{0}$ and $\mu_{1}$ arise as images under radonifying operator, their characterization assumes the following form.

Proposition 3.2. Let $H$ be a quasi-complete real Hilbert space, let $E$ be a real locally convex topological vector space $E$, and let $T_{0}, T_{1} \in \Lambda(H, E)$ be radonifying. Let $\mu_{j}$ be the centered Gaussian measure on $E$ which extends $T_{j}\left(\gamma_{H}\right)(j=0,1)$. Let $Q_{j}=T_{j} \circ T_{j}^{\prime}$ be the covariance ooperator of $\mu_{j}$ and let $H_{j}$ denote its $R K H S$. Then $\mu_{0} \sim \mu_{1}$ if and only if the following two conditions hold:

(i) As subsets of $E$ we have $H_{0}=H_{1}$;

(iii) The map $Q_{0} x^{\prime} \mapsto Q_{0} x^{\prime}-j_{H_{1}, H_{0}} Q_{1} x^{\prime}\left(x^{\prime} \in E^{\prime}\right)$ extends to a Hilbert-Schmidt operator on $H_{0}$; here $j_{H_{1}, H_{0}}: H_{1} \rightarrow H_{0}$ is the identity map.

\section{Equivalence of Ornstein-Uhlenbeck Processes}

Throughout the rest of the paper, $B$ is a separable real Banach space, $H$ is real Hilbert subspace of $B$.

Consider a $C_{0}$-semigroup $\mathscr{S}=\{S(t)\}_{t \geqslant 0}$ of bounded linear operators on $H$, with generator $A$. We assume that for $t>0$ the linear operator $S(t)$ maps $H$ into $B$. The resulting operator will be denoted by $S_{B}(t)$. We suppose that

$$
\int_{0}^{T}\left\|S_{B}^{*}(t) x^{*}\right\|_{H}^{2} d t<\infty, \quad \forall x^{*} \in B^{*} .
$$


A $B$-valued stochastic process $\xi=\left\{\xi_{t}\right\}_{t \in[0, T]}$ on some probability space $(\Omega, \mathbb{P})$ is called an $B$-valued Ornstein-Uhlenbeck process (with drift $\mathscr{S}$ ) if for all $t, s \in[0, T]$ and $x^{*}, y^{*} \in B^{*}$ (the Banach space dual of $B$ ) we have

$$
\mathbb{E}\left(\left\langle\xi_{t}, x^{*}\right\rangle\left\langle\xi_{s}, y^{*}\right\rangle\right)=\int_{0}^{t \wedge s}\left[S^{*}(t-u) i^{*} x^{*}, S^{*}(s-u) i^{*} x^{*}\right]_{H} d u .
$$

Notice that some authors call a process with this property a $B$-valued Ornstein-Uhlenbeck process with drift $A$.

Associated with $\mathscr{S}$ is a positive symmetric operator $Q_{T} \in \Lambda(H)$ defined by

$$
Q_{T} h:=\int_{0}^{T} S(s) S^{*}(s) h d s \quad(h \in H) .
$$

The RKHS associated with $Q_{T}$ will be denoted by $H_{T}$, and the inclusion mapping of $H_{T}$ into $H$ by $i_{T}$. Thus, $Q_{T}=i_{T} \circ i_{T}^{*}$. We assume that $H_{T} \subset B$ and denote by $j_{T}$ the corresponding inclusion map of $H_{T}$ into $B$.

It is easy to see (cf. Proposition 2.3 in [2]) that the existence of a $B$-valued OrnsteinUhlenbeck process with drift $\mathscr{S}$ implies that the inclusion map $j_{T}: H_{T} \hookrightarrow B$ is radonifying. By the results in [2] this implies the existence of an Ornstein-Uhlenbeck measure $\nu$ with drift $\mathscr{S}$ on the product $\sigma$-algebra of the (quasi-complete) product space $B^{[0, T]}$, i.e. a probability measure $\nu$ such that the canonical process $\omega \mapsto \omega(t)$ on the probability space $\left(B^{[0, T]}, \nu\right)$ is a $B$-valued Ornstein-Uhlenbeck process with drift $\mathscr{S}$. It turns out that the measure $\nu$ arises as the image under the (radonifying) map $k \circ \Phi: L^{2}([0, T] ; H) \rightarrow B^{[0, T]}$, where $k: C_{0}([0, T], B) \rightarrow B^{[0, T]}$ is the natural inclusion map and $\Phi: L^{2}([0, T] ; H) \rightarrow C_{0}([0, T], B)$ is the convolution operator defined by

$$
(\Phi f)(t)=\int_{0}^{t} S_{B}(t-s) f(s) d s \quad\left(t \in[0, T], f \in L^{2}([0, T] ; H)\right) .
$$

It is further shown in [2] that the process $\xi$ has a continuous version if $\mathscr{S}$ is an analytic semigroup. In this case, the Ornstein-Uhlenbeck measure $\nu$ associated with $\xi$ may be realized on $C_{0}([0, T] ; B)$ as well. Moreover, in this case $H_{T}$ is equal to the real interpolation space $D_{A}\left(\frac{1}{2}, 2\right)=(D(A), H)_{\left.\frac{1}{2}, 2\right)}$, with equivalent norms.

In order to capture the general case and the analytic case in one framework, throughout the rest of this section we will make the following

Assumption 4.1. Let $H$ and $B$ be as before. Given are two $C_{0}$-semigroups $\mathscr{S}_{0}$ and $\mathscr{S}_{1}$ on $H$ satisfying the assumptions listed earlier in this section, a real quasi-complete locally convex topological vector space $E$, and an injective continuous linear map $\kappa$ from $C_{0}([0, T] ; B)$ into $E$ such that the operators $\kappa \circ \Phi_{0}$ and $\kappa \circ \Phi_{1}$ are radonifying.

Here, $\Phi_{j}: L^{2}([0, T] ; H) \rightarrow C_{0}([0, T] ; B)$ is the convolution operator associated with $\mathscr{S}_{j}$, as defined by $(4.1)$.

Example 4.2. From the above discussion we see that Assumption 4.1 is fulfilled in the following situations:

(i) If the inclusion $j_{T}: H_{T} \hookrightarrow B$ is radonifying we may take $E=B^{[0, T]}$ and $\kappa=k$, the natural inclusion map from $C_{0}([0, T] ; B)$ into $B^{[0, T]}$.

(ii) If $\mathscr{S}$ is analytic and the inclusion $D_{A}\left(\frac{1}{2}, 2\right) \hookrightarrow B$ is radonifying we may take $E=$ $C_{0}([0, T] ; B)$ and $\kappa=I$, the identity operator on $C_{0}([0, T] ; B)$. Alternatively one may take $E=L^{2}([0, T], B)$ with $\kappa: C_{0}([0, T] ; B) \hookrightarrow L^{2}([0, T], B)$ the inclusion map. 
(iii) If $H=B$ and $S_{j}(t)$ is Hilbert-Schmidt for all $t \in(0, T]$, with

$$
\int_{0}^{T}\left\|S_{j}(t)\right\|_{\mathscr{L}_{2}(H)}^{2} d t<\infty, \quad j=0,1
$$

then $\Phi_{0}$ and $\Phi_{1}$ are Hilbert-Schmidt operators on $L^{2}([0, T] ; H)$ and hence radonifying. We then may take $E=L^{2}([0, T] ; H)$ and $\kappa=I$, the identity mapping on $L^{2}([0, T] ; H)$. This is the situation considered by Peszat [9].

We define $\nu_{j}:=\left(\kappa \circ \Phi_{j}\right)\left(\gamma_{L^{2}([0, T] ; H)}\right)(j=0,1)$. Being centered Gaussian measures on $E, \nu_{0}$ and $\nu_{1}$ are either equivalent or singular. Recalling [3, Appendix B] that $\Phi_{0}$ and $\Phi_{1}$ are injective, we have the following necessary and sufficient conditions for equivalence of the measures $\nu_{0}$ and $\nu_{1}$ :

Theorem 4.3. We have equivalence $\nu_{0} \sim \nu_{1}$ if and only if the following two conditions hold:

(i) $\operatorname{range} \Phi_{0}=\operatorname{range} \Phi_{1}$;

(ii) The operator $\Phi_{1}^{-1} \Phi_{0}-\left(\Phi_{0}^{-1} \Phi_{1}\right)^{*}$ is Hilbert-Schmidt on $L^{2}([0, T] ; H)$.

Proof. Let $R_{j}$ denote the covariance operator of the measure $\nu_{j}$. By Proposition 3.1 the RKHS $H_{j}$ of $R_{j}$ equals range $\left(\kappa \circ \Phi_{j}\right)$. By the injectivity of $\kappa$ we therefore have $H_{0}=H_{1}$ if and only if range $\Phi_{0}=\operatorname{range} \Phi_{1}$.

It remains to prove that, under the assumption range $\Phi_{0}=$ range $\Phi_{1}$, the operator $V$ defined by $R_{0} \phi \mapsto R_{0} \phi-R_{1} \phi\left(\phi \in E^{\prime}\right)$ is Hilbert-Schmidt on $H_{0}$ (note that we identify $H_{0}$ and $H_{1}$ in order to simplify notation) if and only if $\Phi_{1}^{-1} \Phi_{0}-\left(\Phi_{0}^{-1} \Phi_{1}\right)^{*}$ is Hilbert-Schmidt on $L^{2}([0, T] ; H)$. To see this, notice that for all $\phi \in E^{\prime}$ we have

$$
\begin{aligned}
V\left(\kappa \Phi_{0} \Phi_{0}^{*} \kappa^{*} \phi\right) & =V\left(R_{0} \phi\right)=R_{0} \phi-R_{1} \phi \\
& =\kappa \Phi_{0} \Phi_{0}^{*} \kappa^{*} \phi-\kappa \Phi_{1} \Phi_{1}^{*} \kappa^{*} \phi \\
& =\kappa \Phi_{0}\left(I-\Phi_{0}^{-1} \Phi_{1}\left(\Phi_{0}^{-1} \Phi_{1}\right)^{*}\right) \Phi_{0}^{*} \kappa^{*} \phi \\
& =\kappa \Phi_{0}\left(\Phi_{0}^{-1} \Phi_{1}\left(\Phi_{1}^{-1} \Phi_{0}-\left(\Phi_{0}^{-1} \Phi_{1}\right)^{*}\right)\right) \Phi_{0}^{*} \kappa^{*} \phi
\end{aligned}
$$

Now $\kappa \circ \Phi_{0}$ is unitary as a map from $L^{2}([0, T] ; H)$ onto $H_{0}=$ range $\left(\kappa \circ \Phi_{0}\right)$. Moreover, $\left(\kappa \circ \Phi_{0}\right)^{*}$ has weak ${ }^{*}$-dense range in $L^{2}([0, T] ; H)$ (being the adjoint of an injective operator) and hence dense range in $L^{2}([0, T] ; H)$ (by reflexivity and the Hahn-Banach theorem). From this we see that $V$ is Hilbert-Schmidt if and only if the map

$$
f \mapsto\left(\Phi_{0}^{-1} \Phi_{1}\left(\Phi_{1}^{-1} \Phi_{0}-\left(\Phi_{0}^{-1} \Phi_{1}\right)^{*}\right)\right) f
$$

is Hilbert-Schmidt on $L^{2}([0, T] ; H)$. But $\Phi_{0}^{-1} \Phi_{1}$ is an isomorphism from $L^{2}([0, T] ; H)$ onto $L^{2}([0, T] ; H)$, so that we finally obtain that $V$ is Hilbert-Schmidt if and only if $\Phi_{1}^{-1} \Phi_{0}-$ $\left(\Phi_{0}^{-1} \Phi_{1}\right)^{*}$ is Hilbert-Schmidt on $L^{2}([0, T] ; H)$.

In view of this result it will be important to find conditions under which we have range $\Phi_{0}=$ range $\Phi_{1}$, and to obtain explicit expressions for the operators $\Phi_{1}^{-1} \Phi_{0}$ and $\Phi_{0}^{-1} \Phi_{1}$. This will be achieved in a series of lemmas.

The first of these extends [9, p. 272] and [3, Remark 10.9].

Lemma 4.4. If range $\Phi_{0} \subset$ range $\Phi_{1}$, then $D\left(A_{0}\right) \subset D\left(A_{1}\right)$.

Proof. By assumption for every $f \in L^{2}([0, T] ; H)$ there exists a function $g \in L^{2}([0, T] ; H)$ such that $\Phi_{1} g=\Phi_{0} f$ in $C_{0}([0, T] ; B)$. Taking Laplace transforms, this is equivalent to the statement that for every $f \in L^{2}([0, T] ; H)$ there exists a function $g \in L^{2}([0, T] ; H)$ such that

$$
R\left(\lambda, A_{1}\right)\left(\Lambda g(\lambda)-e^{-\lambda T} \Phi_{1} g(T)\right)=R\left(\lambda, A_{0}\right)\left(\Lambda f(\lambda)-e^{-\lambda T} \Phi_{0} f(T)\right)
$$


for all large enough positive real numbers $\lambda$; here $\Lambda$ denotes the Laplace transform. Fix $M>0$ and $\omega \in \mathbb{R}$ such that $\max \left\{\left\|S_{0}(t)\right\|,\left\|S_{1}(t)\right\|\right\} \leqslant M e^{\omega t}$ for all $t \geqslant 0$, and fix $\lambda_{0}>\omega$ such that

$$
\frac{M}{\lambda_{0}-\omega}<T
$$

For $\alpha \in \mathbb{R}$ and $h \in H$ let $f_{\alpha, h} \in L^{2}([0, T] ; H)$ be defined by $f_{\alpha, h}(t)=e^{\alpha t} \otimes h$. If $\alpha$ satisfies $\omega<\alpha<\lambda_{0}$, then

$$
\begin{aligned}
\Lambda f_{\alpha, h}\left(\lambda_{0}\right)-e^{-\lambda_{0} T} \Phi_{0} f_{\alpha, h}(T) & =\frac{1-e^{-\left(\lambda_{0}-\alpha\right) T}}{\lambda_{0}-\alpha}\left(h-e^{-\left(\lambda_{0}-\alpha\right) T} \frac{\lambda_{0}-\alpha}{1-e^{-\left(\lambda_{0}-\alpha\right) T}} \int_{0}^{T} e^{-\alpha t} S_{0}(t) h d t\right) \\
& =: \frac{1-e^{-\left(\lambda_{0}-\alpha\right) T}}{\lambda_{0}-\alpha}\left(I-L_{\alpha}\right) h .
\end{aligned}
$$

Since

$$
\left\|\int_{0}^{T} e^{-\alpha t} S_{0}(t) h d t\right\| \leqslant M\|h\| \int_{0}^{T} e^{-(\alpha-\omega) t} d t=M\|h\| \frac{1-e^{-(\alpha-\omega) T}}{\alpha-\omega},
$$

the choice of $\lambda_{0}$ shows that the operator $I-L_{\alpha}$ is invertible if $\lambda_{0}-\alpha$ is small enough.

It follows that

$$
\left\{R\left(\lambda, A_{0}\right)\left(\Lambda f(\lambda)-e^{-\lambda T} \Phi_{0} f(T)\right): f \in L^{2}([0, T] ; H), \lambda>\omega\right\}=D\left(A_{0}\right) .
$$

Hence, in view of 4.2 for all $h \in D\left(A_{0}\right)$ there exists $g \in L^{2}([0, T] ; H)$ such that

$$
R\left(\lambda, A_{1}\right)\left(\Lambda g(\lambda)-e^{-\lambda T} \Phi_{1} g(T)\right)=h .
$$

As the left-hand side defines an element of $D\left(A_{1}\right)$, this proves that $D\left(A_{0}\right) \subset D\left(A_{1}\right)$.

The following lemma gives a partial representation of the operator $\Phi_{1}^{-1} \Phi_{0}$.

Lemma 4.5. Suppose $D\left(A_{0}\right) \subset D\left(A_{1}\right)$ and let $f \in C\left([0, T] ; D\left(A_{0}\right)\right)$. Then for all $t \in[0, T]$,

$$
\Phi_{0} f(t)-\Phi_{1} f(t)=\left(\Phi_{1} \int_{0}^{\cdot}\left(A_{0}-A_{1}\right) S_{0}(\cdot-s) f(s) d s\right)(t) .
$$

Proof. A direct computation gives

$$
\begin{aligned}
\left(\Phi_{1}\right. & \left.\int_{0}\left(A_{0}-A_{1}\right) S_{0}(\cdot-s) f(s) d s\right)(t) \\
& =\int_{0}^{t} \int_{0}^{\tau} S_{1}(t-\tau)\left(A_{0}-A_{1}\right) S_{0}(\tau-s) f(s) d s d \tau \\
& =\int_{0}^{t} \int_{s}^{t} S_{1}(t-\tau)\left(A_{0}-A_{1}\right) S_{0}(\tau-s) f(s) d \tau d s \\
& =\int_{0}^{t} \int_{s}^{t}\left(\frac{d}{d \tau} S_{1}(t-\tau) S_{0}(\tau-s)\right) f(s) d \tau d s \\
& =\int_{0}^{t}\left(S_{0}(t-s)-S_{1}(t-s)\right) f(s) d s \\
& =\Phi_{0} f(t)-\Phi_{1} f(t) .
\end{aligned}
$$

From this we obtain the following converse of Lemma 4.4: 
Lemma 4.6. Suppose $D\left(A_{0}\right) \subset D\left(A_{1}\right)$. If the operator

$$
f \mapsto \int_{0}^{\cdot}\left(A_{0}-A_{1}\right) S_{0}(\cdot-s) f(s) d s, \quad f \in C\left([0, T] ; D\left(A_{0}\right)\right)
$$

extends to a bounded linear operator from $L^{2}([0, T] ; H)$ into itself, then range $\Phi_{0} \subset$ range $\Phi_{1}$.

Proof. Denote the bounded extension by $\Lambda$. Then by Lemma $4.5, \Phi_{0}=\Phi_{1}(I+\Lambda)$ on the dense subspace $C\left([0, T] ; D\left(A_{0}\right)\right)$ of $L^{2}([0, T] ; H)$. Therefore this identity extends to all of $L^{2}([0, T] ; H)$, which gives the result.

We are now in a position to prove Theorem 1.1:

Theorem 4.7. The conditions
(i) $D\left(A_{0}\right)=D\left(A_{1}\right)$ and $\int_{0}^{T}\left\|\left(A_{0}-A_{1}\right) S_{0}(s)\right\|_{\mathscr{L}_{2}(H)}^{2} d s<\infty$
(ii) $D\left(A_{0}\right)=D\left(A_{1}\right)$ and $\int_{0}^{T}\left\|\left(A_{1}-A_{0}\right) S_{1}(s)\right\|_{\mathscr{L}_{2}(H)}^{2} d s<\infty$

are equivalent and imply

(iii) $\nu_{0} \sim \nu_{1}$.

Proof. The equivalence of (i) and (ii) follows from Proposition 2.2.

Assume now that (i) (and therefore also (ii)) holds. In view of Theorem 4.3, in order to prove that $\nu_{0} \sim \nu_{1}$ it is enough to show that range $\Phi_{0}=\operatorname{range} \Phi_{1}$ and that both $\Phi_{1}^{-1} \Phi_{0}-I$ and $\Phi_{0}^{-1} \Phi_{1}-I$ are Hilbert-Schmidt on $L^{2}([0, T] ; H)$.

The finiteness of the integrals in (i) and (ii) implies that

$$
\int_{0}^{T}\left\|\left(A_{0}-A_{1}\right) S_{0}(s)\right\| d s<\infty \text { and } \int_{0}^{T}\left\|\left(A_{1}-A_{0}\right) S_{1}(s)\right\| d s<\infty,
$$

and therefore by Young's inequality the operators

$$
f \mapsto \int_{0}^{\cdot}\left(A_{0}-A_{1}\right) S_{0}(\cdot-s) f(s) d s \quad \text { and } \quad f \mapsto \int_{0}^{\cdot}\left(A_{1}-A_{0}\right) S_{1}(\cdot-s) f(s) d s
$$

are bounded on $L^{2}([0, T] ; H)$. Now Lemma 4.6 shows that range $\Phi_{0}=\operatorname{range} \Phi_{1}$.

For all $f \in L^{2}([0, T] ; H)$ and almost all $t \in[0, T]$ we have

$$
\left(\Phi_{1}^{-1} \Phi_{0} f-f\right)(t)=\int_{0}^{t}\left(A_{0}-A_{1}\right) S_{0}(t-s) f(s) d s .
$$

Indeed, for $f \in C\left([0, T] ; D\left(A_{0}\right)\right)$ this follows from Lemma 4.5 and the general case follows from this by a density argument. We conclude that the operator $\Phi_{1}^{-1} \Phi_{0}-I$ can be represented as an integral operator on $L^{2}([0, T] ; H)$ with operator-valued kernel $K(s, t)=\left(A_{0}-A_{1}\right) S_{0}(t-$ $s)$. Since by (i),

$$
\int_{0}^{T} \int_{0}^{T}\|K(s, t)\|_{\mathscr{L}_{2}(H)}^{2} d s d t<\infty
$$

the operator-valued version of Mercer's theorem [7] now implies that $\Phi_{1}^{-1} \Phi_{0}-I$ is HilbertSchmidt. The same argument shows that also $\Phi_{0}^{-1} \Phi_{1}-I$ is Hilbert-Schmidt.

Theorem 4.7 admits a partial converse, for which we need the following simple observation.

Lemma 4.8. Suppose $\phi:(0, T) \rightarrow \mathbb{R}_{+}$is a measurable function with the following property: there exists a constant $M>0$ such that for all $s, t \in(0, T)$ with $s \leqslant t$ we have $\phi(s) \geqslant M \phi(t)$. If

$$
\int_{0}^{T} \int_{0}^{t} \phi(s) d s d t<\infty
$$


then also

$$
\int_{0}^{T} \phi(s) d s<\infty
$$

Proof. By the finiteness of the double integral, there exists $\delta \in(0, T)$ such that $\int_{0}^{\delta} \phi(s) d s<$ $\infty$. But from

$$
\int_{0}^{T} \int_{0}^{t} \phi(s) d s d t \geqslant \int_{\delta}^{T} \int_{0}^{t} \phi(s) d s d t \geqslant M \int_{\delta}^{T} \int_{0}^{t} \phi(t) d s d t \geqslant \delta M \int_{\delta}^{T} \phi(t) d t
$$

we conclude that also $\int_{\delta}^{T} \phi(t) d t<\infty$.

Now we are in a position to prove Theorem 1.2:

Theorem 4.9. If $\nu_{0} \sim \nu_{1}$, then $D\left(A_{0}\right)=D\left(A_{1}\right)$. If furthermore for each $t \in(0, T]$ the operator $\left(A_{0}-A_{1}\right) S_{0}(t)$ has a bounded extension to $H$ such that

$$
\int_{0}^{T}\left\|\left(A_{0}-A_{1}\right) S_{0}(t)\right\| d t<\infty
$$

then for each $t \in(0, T]$ the operators $\left(A_{0}-A_{1}\right) S_{0}(t)$ and $\left(A_{1}-A_{0}\right) S_{1}(t)$ extend to HilbertSchmidt operators on $H$ and

$$
\int_{0}^{T}\left\|\left(A_{0}-A_{1}\right) S_{0}(s)\right\|_{\mathscr{L}_{2}(H)}^{2} d s<\infty \quad \text { and } \quad \int_{0}^{T}\left\|\left(A_{1}-A_{0}\right) S_{1}(s)\right\|_{\mathscr{L}_{2}(H)}^{2} d s<\infty .
$$

Proof. By Theorem 4.3, $\nu_{0} \sim \nu_{1}$ implies that range $\Phi_{0}=$ range $\Phi_{1}$. Hence equality of the domains $D\left(A_{0}\right)=D\left(A_{1}\right)$ follows from Lemma 4.4 .

By (4.3), Young's inequality and Lemma 4.5, for all $f \in L^{2}([0, T] ; H)$ we have

$$
\Phi_{1}^{-1} \Phi_{0} f-f=\int_{0}^{\cdot}\left(A_{0}-A_{1}\right) S_{0}(\cdot-s) f(s) d s .
$$

Further, by (4.3) and Proposition 2.1, every operator $\left(A_{1}-A_{0}\right) S_{1}(t)$ has a bounded extension to $H$ and we have

$$
\int_{0}^{T}\left\|\left(A_{1}-A_{0}\right) S_{1}(t)\right\| d t<\infty .
$$

Using this, it is easy to check that

$$
\left(\Phi_{0}^{-1} \Phi_{1}\right)^{*} f-f=\int_{.}^{T}\left(\left(A_{1}-A_{0}\right) S_{1}(\cdot-s)\right)^{*} f(s) d s
$$

for all $f \in L^{2}([0, T] ; H)$. Hence,

$$
\begin{aligned}
& \left(\Phi_{1}^{-1} \Phi_{0}-\left(\Phi_{0}^{-1} \Phi_{1}\right)^{*}\right) f(t) \\
& \quad=\int_{0}^{T} \chi_{(0, t)}(s)\left(A_{0}-A_{1}\right) S_{1}(t-s) f(s)+\chi_{(t, T)}(s)\left(\left(A_{1}-A_{0}\right) S_{1}(t-s)\right)^{*} f(s) d s
\end{aligned}
$$

for almost $t \in[0, T]$ and all $f \in L^{2}([0, T] ; H)$. Since $\Phi_{1}^{-1} \Phi_{0}-\left(\Phi_{0}^{-1} \Phi_{1}\right)^{*}$ is Hilbert-Schmidt by Theorem 4.3, Mercer's theorem implies that

$$
\int_{0}^{T} \int_{0}^{T} \chi_{(0, t)}(s)\left\|\left(A_{0}-A_{1}\right) S_{1}(t-s)\right\|_{\mathscr{L}_{2}(H)}^{2}+\chi_{(t, T)}(s)\left\|\left(\left(A_{1}-A_{0}\right) S_{1}(t-s)\right)^{*}\right\|_{\mathscr{L}_{2}(H)}^{2} d s d t<\infty .
$$


Clearly, this holds if and only if both

$$
\begin{aligned}
\int_{0}^{T} \int_{t}^{T}\left\|\left(\left(A_{1}-A_{0}\right) S_{1}(t-s)\right)^{*}\right\|_{\mathscr{L}_{2}(H)}^{2} d s d t & =\int_{0}^{T} \int_{0}^{T-t}\left\|\left(A_{0}-A_{1}\right) S_{0}(s)\right\|_{\mathscr{L}_{2}(H)}^{2} d s d t \\
& =\int_{0}^{T} \int_{0}^{t}\left\|\left(A_{0}-A_{1}\right) S_{0}(s)\right\|_{\mathscr{L}_{2}(H)}^{2} d s d t<\infty
\end{aligned}
$$

and

$$
\int_{0}^{T} \int_{0}^{t}\left\|\left(A_{0}-A_{1}\right) S_{1}(s)\right\|_{\mathscr{L}_{2}(H)}^{2} d s d t<\infty .
$$

By Lemma 4.8 applied to the functions

$$
\phi_{j}(s):=\left\|\left(A_{0}-A_{1}\right) S_{j}(s)\right\|_{\mathscr{L}_{2}(H)}^{2},
$$

taking $M_{j}:=\sup _{s \in[0, T]}\left\|S_{j}(s)\right\|^{2}$, these two conditions are equivalent to (4.4).

Theorems 4.7 and 4.9 can be summarized as follows:

Corollary 4.10. The following are equivalent:
(i) $D\left(A_{0}\right)=D\left(A_{1}\right)$ and $\int_{0}^{T}\left\|\left(A_{0}-A_{1}\right) S_{0}(s)\right\|_{\mathscr{L}_{2}(H)}^{2} d s<\infty$;
(ii) $D\left(A_{0}\right)=D\left(A_{1}\right)$ and $\int_{0}^{T}\left\|\left(A_{1}-A_{0}\right) S_{1}(s)\right\|_{\mathscr{L}_{2}(H)}^{2} d s<\infty$;
(iii) $\nu_{0} \sim \nu_{1}$ and $\int_{0}^{T}\left\|\left(A_{0}-A_{1}\right) S_{0}(s)\right\| d s<\infty$;
(iv) $\nu_{0} \sim \nu_{1}$ and $\int_{0}^{T}\left\|\left(A_{1}-A_{0}\right) S_{1}(s)\right\| d s<\infty$.

If $A_{0}=0$, then this reduces to:

Corollary 4.11. If $A_{0}=0$ the following assertions are equivalent:

(i) $A_{1}$ is bounded and Hilbert-Schmidt;

(ii) $\nu_{0} \sim \nu_{1}$.

\section{Remarks.}

(i) We have only considered equivalence of centered Ornstein-Uhlenbeck measures $\nu$ on $E$. These measures can be thought of as representing Ornstein-Uhlenbeck processes starting at 0 . In a similay way an Ornstein-Uhlenbeck processes starting at some $h \in H$ can be represented by a Gaussian measure $\nu^{h}$ on $E$ with mean $\kappa(S(T) h)$ and whose covariance equals the covariance of the corresponding centered OrnsteinUhlenbeck measure. As in [9] it is easy to see that equivalence $\nu_{0} \sim \nu_{1}$ implies $\nu_{0}^{(h)} \sim \nu_{1}^{(h)}$ for all $h \in H$. In fact, by $(2.1)$ we have

$$
S_{1}(T) h-S_{0}(T) h=\int_{0}^{T} S(T-s) \psi(s) d s,
$$

where $\psi:=\left(A_{1}-A_{0}\right) S_{1}(\cdot) h \in L^{2}([0, T] ; H)$ by (4.4). Hence by [3, Corollary B.7], $\kappa\left(S_{1}(T) h-S_{0}(T) h\right)$ belongs to the common RKHS of the measures $\nu_{0}^{(h)}$ and $\nu_{1}^{(h)}$, which shows that condition (ii) of Proposition 3.2 is satisfied.

(ii) Notice that the conditions for equivalence we have obtained are intrinsic, in the sense that they do not refer to the space $B$ but only to $H$ and the semigroups $\mathscr{S}_{0}$ and $\mathscr{S}_{1}$. Furthermore they are independent of $T$.

Acknowledgement - The authors are grateful to Professor Jerzy Zabczyk for helpful discussions concerning this work. 


\section{REFERENCES}

[1] P. Baxendale, Gaussian measures on function spaces, Amer. J. Math. 98 (1976), 891-952.

[2] Z. Brzeźniak and J.M.A.M. van Neerven, Banach space-valued Ornstein-Uhlenbeck processes indexed by the circle, to appear in: L. Weis (ed.): Proceedings of the Conference of Evolution Equations and their Applications, Bad Herrenalb 1998. Birkhauser Verlag.

[3] G. Da Prato and J. Zabczyk, "Stochastic Equations in Infinite Dimensions", Encyclopedia of Mathematics and Its Applications, Vol. 44, Cambridge University Press, Cambridge, 1992.

[4] E. Hille and R.S. Phillips, "Functional Analysis and Semi-Groups", Amer. Math. Soc. Colloq. Publ., Vol. 31 (revised edition), Providence, R.I., 1957.

[5] S.M. Kozlov, Equivalence of measures for Itô's partial differential equations, Vestnik Moskov. Univ. Ser. I Math. Mekh. 4 (1977), 147-152 (Russian).

[6] H.H. Kuo, "Gaussian Measures in Banach Spaces", SLNM 463, Springer-Verlag, 1975.

[7] W. Mlak, "Hilbert Spaces and Operator Theory", Mathematics and Its Applications, Vol. 51, Kluwer Academic Publishers and PWN - Polish Scientific Publishers, 1991.

[8] A. Pazy, "Semigroups of Linear Operators and Applications to Partial Differential Equations", Applied Mathematical Sciences, Springer-Verlag, Berlin-Heidelberg-New York, 1983.

[9] Sz. Peszat, Law equivalence of solutions of some linear stochastic equations in Hilbert spaces, Studia Mathematica 101 (1992), 269-284.

[10] R. Ramer, On nonlinear transformations of Gaussian measures, J. Funct. Anal. 15 (1974), 166-187.

[11] H. Röckle, "Abstract Wiener Spaces, Infinite-Dimensional Gaussian Processes and Applications", Ph.D. Dissertation, Ruhr-Univerisität Bochum (1993).

[12] L. Schwartz, Sous-espaces Hilbertiens d'espaces vectoriels topologiques et noyaux associés, J. d'Analyse Math. 13 (1964), 11-256.

[13] L. Schwartz, "Radon Measures on Arbitrary Topological Vector Spaces", Oxford University Press, Oxford, 1973.

[14] N.N. Vakhania and V.I. Tarieladze, On singularity and equivalence of Gaussian measures, in: M.M. Rao (Ed.), "Real and Stochastic Analysis: Recent Advances", pp. 367-389, CRC Press, Boca Raton-New York, 1997.

[15] N.N. Vakhania, V.I. Tarieladze, and S.A. Chobanyan, "Probability Distributions on Banach Spaces", D. Reidel Publishing Company, Dordrecht-Boston-Lancaster-Tokyo, 1987.

Department of Mathematics, The Univeristy of Hull, Hull HU6 7RX, England

E-mail address: Z.Brzezniak@maths.hull.ac.uk

Department of Mathematics, Delft University of Technology, PO Box 5031, 2600 Ga Delft, The Netherlands

E-mail address: J.vanNeerven@twi.tudelft.nl 